\title{
Immature Platelet Fraction and COVID-19: Maturing prognostic links!
}

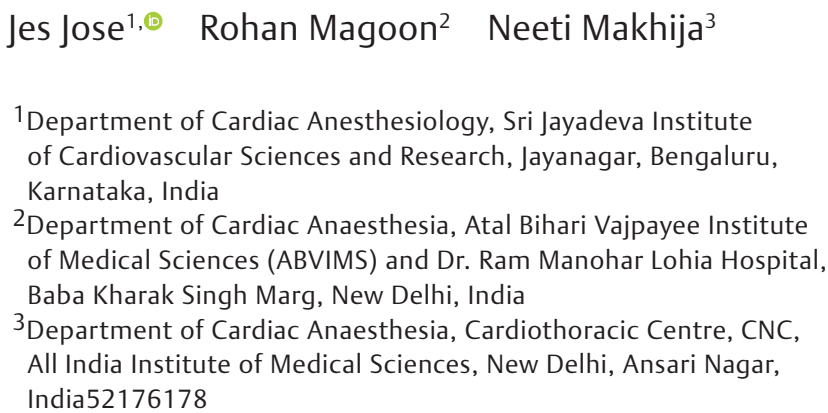

Jes Jose ${ }^{1, \odot}$ Rohan Magoon ${ }^{2}$ Neeti Makhija ${ }^{3}$

${ }^{1}$ Department of Cardiac Anesthesiology, Sri Jayadeva Institute of Cardiovascular Sciences and Research, Jayanagar, Bengaluru, Karnataka, India

2Department of Cardiac Anaesthesia, Atal Bihari Vajpayee Institute of Medical Sciences (ABVIMS) and Dr. Ram Manohar Lohia Hospital, Baba Kharak Singh Marg, New Delhi, India

${ }^{3}$ Department of Cardiac Anaesthesia, Cardiothoracic Centre, CNC, All India Institute of Medical Sciences, New Delhi, Ansari Nagar, India52176178

\begin{abstract}
Address for correspondence Neeti Makhija, MD, Department of Cardiac Anaesthesia, Room No. 9, 7th Floor, Cardiothoracic Centre, All India Institute of Medical Sciences, Ansari Nagar, New Delhi, 110029, India (e-mail: neetimakhija@hotmail.com).
\end{abstract}

\author{
Abstract \\ Keywords \\ - COVID-19 \\ - Immature platelet \\ fraction \\ - Platelets \\ - Prognostication \\ - SARS-CoV-2 infection \\ - Sepsis \\ - Thromboembolic \\ events
}

Prompted by the combination of an ever-evolving comprehension of the platelet activation as a pivotal perpetuator of an ongoing systemic inflammatory process and an encouraging literature on the prognostic role of immature platelet fraction (IPF) in septic and prothrombotic settings, we present an elaborated account of the possible prognostic links between IPF and Coronavirus disease 2019 (COVID-19).

\section{Introduction}

Platelets (miniature anucleate cells) are peculiarly intriguing with regard to the diversity of their pathobiophysiological roles, extending much beyond hemostasis-thrombosis to the evolving reputation as immune mediators, staging the complex interactions between the immune system, leukocytes, endothelial cells, and the clotting cascade..$^{1,2}$

As an extension of the same, the severe acute respiratory syndrome Coronavirus 2 (SARS-CoV-2) and platelet communion continues to captivate an increasing attention of the fraternity. This becomes all the more pertinent amid an enhanced acknowledgment of the vascular and coagulation links of the ongoing Coronavirus disease 2019
(COVID-19)-related endotheliitis, which is the cornerstone of lethal thromboembolic consequences. ${ }^{3-5}$

\section{Background}

While the platelet-related parameters such as mean platelet volume (MPV) and MPV/platelet count ratio have been investigated for their ability to augment the differentiation of COVID-19 from the influenza pneumonia and for the severity prediction in COVID-19 with promising results, the immature platelet fraction (IPF) has received much lesser scrutiny as the platelet activation readouts of prognostic importance in COVID-19. ${ }^{6,7}$ published online August 5, 2021
DOI https://doi.org/ 10.1055/s-0041-1732836 ISSN 2457-0206 (c) 2021. Official Publication of The Simulation Society (TSS), accredited by International Society of Cardiovascular Ultrasound (ISCU).

This is an open access article published by Thieme under the terms of the Creative Commons Attribution-NonDerivative-NonCommercial-License, permitting copying and reproduction so long as the original work is given appropriate credit. Contents may not be used for commercial purposes, or adapted, remixed, transformed or built upon. (https://creativecommons.org/licenses/by-nc-nd/4.0/). Thieme Medical and Scientific Publishers Pvt. Ltd. A-12, 2nd Floor, Sector 2, Noida-201301 UP, India 
Herein, IPF denotes the proportion of the total platelet pool constituted by the young immature reticulated cells and constitutes an important, inexpensive, an automated hematological marker of an accentuated platelet turnover. ${ }^{1,7}$ Quite remarkably, the combined account of the initial encouraging literature on the prognostic role of IPF, in septic and prothrombotic clinical settings, endorses a strong case for exploring the predictive abilities in COVID-19. ${ }^{7-12}$

\section{IPF in a Septic Setting}

$\mathrm{Wu}$ and colleagues demonstrated the prognostic role of reticulated platelets percentage (RP\%), wherein a higher $\mathrm{RP} \%$ emerged as a major predictor of mortality with an area under the curve of 0.867 in their prospective evaluation of a septic cohort. ${ }^{8}$ The depiction of the predictive value was robust to a subsequent multilogistic regression analysis. Considering the pivotal role of microcirculatory alterations and coagulation disturbances in sepsis, Muronoi et al delineated an extended role of the admission-IPF in predicting subsequent disseminated intravascular coagulation (DIC) and poor outcomes. ${ }^{9}$

Interestingly, Park and the group outlined the highest sensitivity and specificity of IPF compared with biomarkers such as lactate, C-reactive protein (CRP), and procalcitonin, in distinguishing the septic and nonseptic population while studying 312 general ward patients. ${ }^{10}$ However, the predictive value of IPF declined in delineating the sepsis severity in their study.<sup $>10$

\section{IPF in a Prothrombotic Setting}

In context of prothrombotic setting, platelet activation has been extensively studied in acute coronary syndromes (ACS) and strokes. The immature RPs, newly released into the circulation from the marrow by the megakaryocytes, possess highly dense granular and residual messenger ribonucleic acid (mRNA) content. These immature forms are proposed to demonstrate an elevated hyperactivity and an enhanced thrombotic propensity, as delineated in their association with thrombotic events in cardiovascular and cerebrovascular settings. ${ }^{1,7}$ IPF has emerged as an independent predictor of major adverse cardiovascular event and cardiovascular mortality in the ACS subset.11,12

At the same time, the recent literature on the role of RP\% as a predictor of perioperative myocardial injury (PMI) is noteworthy, particularly when PMI is described to be associated with poor outcomes. ${ }^{13}$ Meershoek and colleagues conducted an observational cohort study comprising 2289 patients undergoing major noncardiac surgery wherein the incidence of PMI (troponin I $>0.06 \mathrm{mg} / \mathrm{L}$ ) and the 30-day mortality was significantly higher for subjects with a preoperative RP $\geq$ $2.82 \%$ ( $36.1 \%$; $8.6 \%$, respectively) in contrast to those demonstrating a normal preoperative RP percentage (28.3\%; 3.6\%, respectively)..$^{14}$

\section{Extrapolating the Links to COVID-19}

While a COVID-19 infection also incurs a considerable prothrombotic risk, the seminal work of Cohen and colleagues on the prospective comparison of IPF among 47 COVID-19 patients, 100 acute myocardial infarction (AMI) patients, and 64 stable cardiovascular disease predisposed patients, is impressive. ${ }^{4,5,7,15-18}$ They described that IPF on admission was significantly higher in the COVID-19 cohort (4.8\%; interquartile range [IQR]: 3.4-6.9) compared with the stable cohort (3.5\%; IQR: 2.7-5.1) and comparable to the AMI cohort (4.55\%; IQR: 3.0-6.75). Similarly, the maximal in-hospital IPF was also substantially higher for the COVID-19 and the AMI group of patients in contrast to the stable cardiovascular patients. ${ }^{7}$

Although their study does not suggest an association of IPF with COVID-19 disease severity, a small sample size of COVID-19 patients with severe disease could have very well precluded the power of the study to assess the same. Nevertheless, given the fact that the three groups were well-matched for their cardiovascular predisposition, a quantitative estimation of enhanced platelet turnover can assist in the management of COVID-19 patients, particularly backed by a motivated exploration of thromboembolic events in COVID-19 patients with an elevated IPF.?

Talking primarily of acute respiratory distress syndrome (ARDS, also a severe complication of sepsis), autopsies of patients succumbing to ARDS have revealed accentuated platelet accumulation in the pulmonary vasculature..$^{19}$ Alongside the demonstration of platelet activation in the bronchoalveolar lavage of ARDS patients, there is understandably an enhanced likelihood of encouraging results emerging from further exploration of the prognostic links between IPF and COVID-19.20

\section{Discussion}

Several additional factors surface on a closer evaluation of this research area:

(i). IPF or RP\% is a readily available parameter subject to flow cytometric or hematological analysis, given the large size, high granularity, and ribonucleic acid content (augmenting fluorescence signal) of these immature forms and often considered superior to the MPV as a platelet immaturity index. ${ }^{1}$

(ii). Moreover, the intriguing combination of thrombocytopenia and prothrombotic events in COVID-19 raises a very likelihood of platelet hyperactivity being at the heart of the matter. ${ }^{7}$

(iii). Ahead of the prognostic relevance, IPF and related indices have also been outlined to be predictors of antiplatelet therapy response. ${ }^{21}$

(iv). Recognition of IPF-based high risk of prothrombotic events can potentially assist in individualizing the antithrombotic regimen in COVID-19. 


\section{Conclusion}

The aforementioned discussion suggests a possible role of cost-effective platelet activation parameters like IPF in parsimonious prognostication of COVID-19, with the subsequent risk-based implementation of optimally individualized management scheme.

\section{Authors' Contributions}

JJ: conceptualization and analysis.

RM: conceptualizing and writing the original draft.

NM: supervising, and reviewing and editing the manuscript.

\section{Ethics Approval and Consent to Participate Not applicable.}

\section{Consent for Publication}

All authors approve the contents of this paper for publication.

\section{Availability of Data and Material Not applicable.}

\section{Funding}

None.

\section{Competing Interests}

The authors declare that they have no competing interests.

\section{Conflict of Interest}

We do not have any conflict of interest, or any commercial or financial interest in this material, and agree to abide by the rules of your journal regarding publication of this article.

\section{References}

1 Schmoeller D, Picarelli MM, Paz Munhoz T, Poli de Figueiredo CE, Staub HL. Mean platelet volume and immature platelet fraction in autoimmune disorders. Front Med (Lausanne) 2017;4:146

2 Dey S, Kashav R, Kohli JK, et al. Systemic immune-inflammation index predicts poor outcome after elective off-pump CABG: a retrospective, single-center study. J Cardiothorac Vasc Anesth 2020;(e-pub ahead of print). doi: 10.1053/j. jvca.2020.09.092

3 Kuchi Bhotla H, Kaul T, Balasubramanian B, et al. Platelets to surrogate lung inflammation in COVID-19 patients. Med Hypotheses 2020;143:110098

4 Magoon R, ItiShri, Kohli JK, Kashav R. Inhaled Milrinone for sick COVID-19 cohort: a pathophysiology driven hypothesis. Med Hypotheses 2020;(e-pub ahead of print). doi: 10.1016/j. mehy.2020.110441

5 Magoon R. Pulmonary vasculature in COVID-19: mechanism to monitoring. Korean J Anesthesiol 2020;(e-pub ahead of print). doi: $10.4097 / \mathrm{kja} .20536$
6 Ozcelik N, Ozyurt S, Yilmaz Kara B, Gumus A, Sahin U. The value of the platelet count and platelet indices in differentiation of COVID-19 and influenza pneumonia. J Med Virol 2020;(e-pub ahead of print). doi: $10.1002 / \mathrm{jmv} .26645$

7 Cohen A, Harari E, Cipok M, et al. Immature platelets in patients hospitalized with Covid-19. J Thromb Thrombolysis (e-pub ahead of print). doi 10.1007/s11239-020-02290-6

$8 \mathrm{Wu}$ Q, Ren J, Hu D, et al. An elevated percentage of reticulated platelet is associated with increased mortality in septic shock patients. Medicine (Baltimore) 2015;94(19):e814

9 Muronoi T, Koyama K, Nunomiya S, et al. Immature platelet fraction predicts coagulopathy-related platelet consumption and mortality in patients with sepsis. Thromb Res 2016;144:169-175

10 Park SH, Ha SO, Cho YU, Park CJ, Jang S, Hong SB. Immature platelet fraction in septic patients: clinical relevance of immature platelet fraction is limited to the sensitive and accurate discrimination of septic patients from non-septic patients, not to the discrimination of sepsis severity. Ann Lab Med 2016;36(1):1-8

11 Cesari F, Marcucci R, Gori AM, et al. Reticulated platelets predict cardiovascular death in acute coronary syndrome patients. Insights from the AMI-Florence 2 Study. Thromb Haemost 2013;109(5):846-853

12 Ibrahim H, Schutt RC, Hannawi B, DeLao T, Barker CM, Kleiman NS. Association of immature platelets with adverse cardiovascular outcomes. J Am Coll Cardiol 2014;64(20):2122-2129

13 Magoon R, Makhija N, Das D. Perioperative myocardial injury and infarction following non-cardiac surgery: A review of the eclipsed epidemic. Saudi J Anaesth 2020;14(1):91-99

14 Meershoek AJ, Leunissen TC, van Waes JAR, et al. Reticulated platelets as predictor of myocardial injury and 30 day mortality after non-cardiac surgery. Eur J Vasc Endovasc Surg 2020;59(2):309-318

15 Magoon R. COVID-19 related strokes: Pandora's box may open as the p(c)lot thickens! Neurologia 2021;(e-pub ahead of print). doi: 10.1016/j.nrl.2021.03.004

16 Magoon R. Left-ventricular diastolic dysfunction in COVID-19: Opening the Pandora's Box! Korean J Anesthesiol 2021;(e-pub ahead of print). doi: 10.4097/kja.21010

17 Magoon R. The pulmonary circuit dynamics in COVID-19! J Anesth 2021;35(1):161

18 Magoon R. COVID-19 and congenital heart disease: Cardiopulmonary interactions for the worse! Paediatr Anaesth 2020;30(10):1160-1161

19 Greco E, Lupia E, Bosco O, Vizio B, Montrucchio G. Platelets and multi-organ failure in sepsis. Int J Mol Sci 2017;18(10):2200

20 Carvalho AC, DeMarinis S, Scott CF, Silver LD, Schmaier AH, Colman RW. Activation of the contact system of plasma proteolysis in the adult respiratory distress syndrome. J Lab Clin Med 1988;112(2):270-277

21 Stratz C, Bömicke T, Younas I, et al. Comparison of immature platelet count to established predictors of platelet reactivity during thienopyridine therapy. J Am Coll Cardiol 2016;68(3):286-293 